\title{
Itinéraires Itinéraires
}

Littérature, textes, cultures

2009-3 | 2009

Modernités occidentales et extra-occidentales

\section{Aux sources de la modernité marocaine}

\section{Khalid Zekri}

\section{OpenEdition}

\section{Journals}

Édition électronique

URL : http://journals.openedition.org/itineraires/463

DOI : 10.4000/itineraires.463

ISSN : 2427-920X

Éditeur

Pléiade

Édition imprimée

Date de publication : 1 novembre 2009

Pagination : 43-55

ISBN : 978-2-296-10115-9

ISSN : $2100-1340$

Référence électronique

Khalid Zekri, « Aux sources de la modernité marocaine », Itinéraires [En ligne], 2009-3 | 2009, mis en ligne le 23 juin 2014, consulté le 19 avril 2019. URL : http://journals.openedition.org/itineraires/463 DOI : 10.4000/itineraires.463

\section{c) ()요}

Itinéraires est mis à disposition selon les termes de la licence Creative Commons Attribution - Pas d'Utilisation Commerciale - Pas de Modification 4.0 International. 


\title{
Aux sources de la modernité marocaine
}

\begin{abstract}
In Morocco, modernity, before being a literary phenomenon, has first been motivated by the desire to understand why the Arabo-islamic world was behind the Western world. For the Moroccan travelers who were sent to Europe by their sultans, modernization was first associated to military order and to technical and industrial discoveries. Such a discovery has generated a double consciousness which characterizes the period of the fight for independence: close link to tradition on the one hand and strong temptation towards modernity on the other. Such a double consciouness has taken the form, in literature, of the use of the genre of the novel starting in the 1950s.
\end{abstract}

Keywords : Morocco, modernity, travel, Islamic reformism, novel

Mots clés : Maroc, modernité, voyage, réformisme islamique, roman

Disons d'emblée que la notion de modernité renvoie ici à tout mouvement de pensée fondé sur la volonté de rupture avec le passé (ce qui ne signifie pas négation de ce passé). Cette rupture trouve son explication dans le désir de faire accéder l'individu au statut de sujet se définissant et se positionnant par rapport à d'autres sujets. Aussi partielle qu'elle puisse paraître, une telle conception de la modernité touche aux fondements du paradigme généalogique. Elle dérègle l'éthique de la rencontre puisque le sentiment de déracinement qu'elle a engendré, dans le cas du Maroc, a remis en question l'idée de souveraineté et a précarisé les singularités identitaires. Elle a également produit une division dans le commun de la communauté et a engendré, par le même geste, le métissage des identités et l'hybridation des imaginaires qui ont permis de repenser, non sans difficulté, ce commun divisé. C'est ainsi que le « commun de la communauté » a pris parfois des formes d'auto-protection excessives : uniformisation culturelle, fanatisme religieux, replis identitaires. Ce qui retiendra notre attention ici, c'est l'examen de la relative modernisation du Maroc et ses répercussions sur la littérature. Pour cela, un geste " archéologique » s'impose. 


\section{La découverte de la modernité par le voyage}

Les conquêtes coloniales de l'Empire français au Maghreb ont donné lieu à des relations de voyages qui ont largement contribué à informer le Maroc et ses élites sur les fondements éducatifs, militaires, scientifiques, industriels et culturels de la modernité européenne. L'exemple qui semble le plus illustratif à cet égard est le récit de voyage d'une ambassade marocaine chez Louis Philippe, rédigé par Mohammed Ben Abdallah Al-Saffâr Al-Tetwânî sous le titre Rihlat Al-Saffâr ilâ Faranssâ (Voyage d'Al-Saffâr en France, 1845-1846 ${ }^{1}$ ). C'est une relation de voyage qu'il a rédigée en tant que scribe ayant accompagné l'ambassadeur marocain Abdelkader Achaâch dans son voyage diplomatique chez Louis Philippe, en France. La rédaction de ce récit de voyage avait pour finalité de répondre aux questions pressantes que se posaient le sultan Moulay Abderrahmane et son élite à propos de la puissance européenne par rapport au monde musulman. Ces questions concernaient les aspects et les moyens militaires, scientifiques, culturels et éducatifs qui ont consolidé la puissance de l'Europe et plus particulièrement celle de la France. Le champ sémantique de la « nouveauté » constitue ainsi le centre d'intérêt du voyageur dont la croyance, les sentiments, la vision et les orientations se trouvaient parfois mis à l'épreuve. Dans un Maghreb secoué par le colonialisme français imposé aux Algériens, le voyage d'AlSaffâr a inauguré, dans une certaine mesure, une nouvelle vision du monde et un nouveau rapport à l'Europe. La mainmise sur l'Algérie signifiait non seulement une difficulté de communication et de circulation entre certains pays musulmans dont l'unité était essentiellement religieuse, mais aussi une menace d'expansionnisme français sur l'ensemble du Maghreb qui, par ailleurs, n'allait pas tarder à se réaliser progressivement.

En 1844, l'année où l'émir Abdelkader s'est réfugié au Maroc, le général Bugeaud a bombardé les deux ports marocains les plus importants de l'époque, Mogador (Essaouira) et Tanger, afin de paralyser les activités commerciales et punir ainsi les Marocains pour avoir accueilli l'émir Abdelkader. La guerre d'Isly a constitué la preuve flagrante de la puissance impériale française qui a écrasé une armée marocaine désordonnée et mal commandée. C'est dans ce contexte que la France a exigé de Moulay Abderrahmane qu'il envoie, avec empressement, un ambassadeur marocain chez le roi Louis-Philippe pour négocier, d'une part, l'éloignement de l'émir Abdelkader du Maroc et, d'autre part, les changements et les réformes que l'État marocain devait appliquer sur son territoire et, par conséquent, dans sa relation à la France. Sur le plan théologique, la négociation avec un pays

1. Abou Dabi, Dar Swidi Linnachr wa tawzi', 2007. Cette édition est la traduction d'un excellent travail d'annotation, d'établissement et de commentaire fait en anglais par Susan Gilson Miller en 1976 et publié dans son ouvrage Disorienting Encounters : Travels of a Moroccan Scholar in France in 1845-1846. The Voyage of Muhammad As-Saffar, California, University of California Press, 1992. 
non musulman posait des problèmes aux jurisconsultes marocains. Après plusieurs concertations et des divergences d'opinion au sein même de l'élite proche du Palais, le sultan a pris la décision d'envoyer Abdelkader Achaâch comme ambassadeur à Paris. Ce choix a été, semble-t-il, essentiellement motivé par la richesse de celui-ci car il avait pris en charge tous les frais de la mission qui lui a été confiée. Dans une lettre confidentielle datée du 5 novembre 1845 et adressée par Léon Roches (chargé des préparatifs concernant cette ambassade qu'il a accompagnée) à Edme de Chasteau (consul de France à Tanger), l'intention expansionniste de l'Empire français est très claire : il s'agit, pour la France, d'étendre son influence et de dominer plus de territoires que tout autre Empire. L'insistance pour qu'un corps diplomatique marocain se déplace en France obéit à une stratégie dont le but était d'exposer, en direct, la puissance et la modernité de la France pour impressionner et intimider l'Etat marocain. C'est ainsi que les représentants du Maroc avaient découvert les voies ferroviaires, la flotte maritime de Toulon, le Théâtre, la presse, les bibliothèques, la trésorerie, les services fiscaux, les maisons d'édition, les écoles modernes et les habitudes alimentaires en France. La construction d'une image aussi exhaustive des différents aspects de la vie française était au service d'un projet hégémonique qui consistait à exercer l'influence de la France sur le Maroc sans recourir à des interventions militaires, comme cela fut le cas en Algérie. Il était d'abord question de dominer le Maroc en façonnant l'imaginaire de l'élite la plus proche du Palais. Ce façonnement visait la " modernisation de l'élite » par un processus de « collaboration consentie ». Cette stratégie portera ses fruits dès le retour de l'ambassadeur Achaâch au Maroc. L'impact de son séjour à Paris sera immédiat puisqu'il proposera lui-même ses services aux Français par une lettre adressée le 10 mars 1846 à De Chasteau. C'est ainsi que la "révolution au sein du Palais » que prévoyait Léon Roches se réalisa progressivement au détriment des intérêts de l'État marocain. La France orchestra une scénographie de corruption généralisée pour maintenir les hommes de pouvoir marocains sous sa dépendance. C'est alors que l'épistémologie théo-politique du sultan et d'une partie de son élite connaîtra des remises en questions. Les notions d'ordre et de désordre seront introduites dans le lexique militaire et les différents discours de l'État. C'est ce qui explique, par ailleurs, l'intérêt que le sultan Moulay Abderrahmane et ses successeurs accordaient à la consignation par écrit de toutes les informations que le corps diplomatique pouvait recueillir lors de ses visites à des pays européens.

Les récits des voyageurs marocains partis en Europe après AsSaffâr ont été marqués par un sentiment d'infériorité face à la modernité européenne comme on peut le constater dans Al-Rihla Al-Tatwijiya ila 'Assimat Al-Bilad Al-Inglisia (Le Voyage de couronnement à la capitale de la contrée anglaise) d'Al-Hassan Ben Mohamed Al-Rhassal qui est parti à Londres en 1902 dans le cadre d'une mission diplomatique. Cette relation 
de voyage touche aux frontières de l'ouverture et de la fermeture par rapport à l'expérience de l'altérité étant donné que l'État marocain hésitait à l'époque entre le désir de s'ouvrir à l'étranger et de le découvrir (sous les pressions des empires européens et l'impulsion d'une élite moderniste) et le désir de sauvegarder l'indépendance de son territoire (sur les conseils des théologiens musulmans). L'élite marocaine s'est ainsi divisée en deux tendances face aux empires européens : une tendance qui considère l'Europe comme un espace susceptible de participer à l'évolution du Maroc et une autre conservatrice qui range le processus de modernisation dans la catégorie de l'impiété et de l'hérésie. Le point commun entre ces deux tendances s'articule autour du paradigme qui oppose Dar Al-Kufr, qu'on appelle aussi Dar Al-Harb (Terre d'hérésie ou Terre de Guerre) et Dar Al-Islam (Terre d'islam). La tendance de propagande en faveur de la modernité européenne trouve peut-être son illustration la plus extrême chez Al-Hajoui Al-Thaâlibi, un autre voyageur-diplomate marocain. Celui-ci a même soutenu l'Empire français dans son entreprise coloniale au Maroc et ses enfants ont joué un rôle important dans le gouvernement de Ben $\mathrm{Arafa}^{2}$. Al-Hajoui Al-Thaâlibi a relaté son voyage effectué en 1919 en Europe dans un récit intitulé Al-Rihla al-Awrubbiya (Le Voyage européen) dans lequel le libéralisme modéré du voyageur révèle le balancement des Marocains entre la nécessité de mener des réformes et la difficulté de les accomplir. Les questions posées par la Nahda ne peuvent pas trouver, selon lui, leurs réponses dans l'imitation totale de l'Occident ou dans un regard exclusivement orienté vers l'avenir. Il plaide pour une ouverture mesurée, mais qui va parfois jusqu'à la collaboration avec le colonisateur. Il a publié un autre ouvrage intitulé L'Ordre en islam dans lequel il insiste sur la nécessité du renouveau permanent car, selon lui, aucune nation ne peut rester statique puisqu'elle est nécessairement soumise à la loi du changement. Ce qu'il a écrit semble relever du bon sens, mais à le lire de près on constate qu'il reproduit le discours colonialiste selon lequel l'expansionnisme européen a pour but d'aider les indigènes à s'engager sur le chemin du progrès. La transmission d'une intention coloniale «noble » se lit en filigrane dans la relation de voyage d'Al-Hajoui Al-Tha'alibi qui faisait partie de ces multiples courroies de transmission dont l'Empire français avait besoin pour mieux asseoir son pouvoir et son hégémonie " par la paix et la prospérité ».

\section{Réformisme marocain et modernité}

$\mathrm{Au} \mathrm{xx}^{\mathrm{e}}$ siècle, le poète et penseur nationaliste Mohammed Allal Al-Fassi (1910-1974) adoptera les positions du réformisme de l'Orient musulman et tentera une synthèse entre le retour à une identité islamique et l'ouverture

2. Il a été intronisé par la Présidence française comme roi du Maroc en 1955 après la déportation et l'exil forcé de son cousin, le sultan Mohamed Ben Youssef (le roi Mohammed V). 
sur la modernité avec un réajustement constant en fonction des principes de l'islam ${ }^{3}$. Les principes de la Nahda seront donc investis au Maroc par des salafistes nationalistes comme Allal Al-Fassi, qui par ailleurs fait figure de leader, pour permettre aux Marocains de recouvrer leur liberté spoliée par le colonisateur. Le salafisme prône un retour aux prescriptions coraniques et prophétiques en les purifiant des impuretés accumulées tout au long de l'histoire du monde musulman, une éducation islamique authentique, une soumission au représentant de Dieu sur terre avec l'idée de transformer son mode de gouvernance de manière progressive car, pour les salafistes, la liberté de l'individu ne peut être conçue en dehors d'une soumission à Dieu et à son représentant temporel. Le salafisme met également en valeur la rationalité de la pensée islamique, exhorte à la solidarité entre Musulmans et à l'établissement d'une théocratie en s'appuyant sur le Coran et le hadith (les dires et les actions du Prophète Muhammad). Cette théocratie a également pour mission de réformer les systèmes militaire, économique et institutionnel pour garantir la liberté en terre d'islam. C'est ainsi que les réformateurs salafistes parlaient de Choura pour désigner la démocratie et évoquaient al-hall wa l'aqd pour parler de représentativité parlementaire. On perçoit aisément derrière ces principes une volonté de structurer la lutte contre les empires coloniaux à travers le retour aux sources et aux textes fondateurs.

Ce mouvement réformiste a permis, dès son émergence, d'exprimer un peuple à venir en se tournant vers le récit de l'islamo-arabité. La fabulation qui est mise en œuvre est celle de la Umma en tant que communauté religieuse transfrontalière. Le champ de la pensée développée par Alla AlFassi vise à restituer aux Marocains, en tant que peuple arabo-musulman, leur identité nationale et théologico-linguistique. C'est là l'une des carences $\mathrm{du}$ discours du réformiste marocain dont les germes restent encore dans le Maroc d'aujourd'hui. La faille de cette idéologie (notamment chez AlAfghani) réside, entre autres, dans son inscription qui n'a pas su déborder les conjonctures circonstancielles pour atteindre une vision historique plus globale. Autrement dit, les nationalistes marocains ont glorifié le passé arabo-islamique et culpabilisé le présent car, selon eux, les gouvernants ne sont pas seulement entrés en complicité avec les empires européens, mais ont également corrompu le « vrai » islam. Ces principes étaient également investis pour permettre au peuple de recouvrer sa liberté spoliée par le colonisateur. C'est pour cela que la lutte contre le colonialisme français a été faite à travers des discours populistes qui faisaient appel à une reconstruction identitaire à partir de récits fondateurs ancestraux qui, tout en légitimant le projet de reconstruction identitaire, créaient l'illusion d'une conscience nationale renaissante. C'était, entre autres raisons, pour cela que les réformistes-modernistes marocains avaient toujours lutté contre

3. Mohammed Allal Al-Fassi, Défense de la loi islamique, (traduit de l'arabe par Charles Samara), Casablanca, Imprimerie Dar El Beida, 1977. 
l'idée de la modernité comme triomphe de l'universel sur le particulier. Une telle conception de la modernité, issue d'un humanisme universaliste abstrait, était possible dans les premiers moments de la modernisation industrielle quand la Grande-Bretagne, la France et les États-Unis d'Amérique s'étaient identifiés à une forme ou à une autre d'universalisme. C'est ce que la France avait fait en se proclamant détentrice des valeurs universelles issues de la Révolution de juillet 1789. Les missions civilisatrices et la déclaration des Droits de l'Homme étaient des prétextes, parmi d'autres, pour légitimer les expéditions coloniales. Et ce n'était pas un hasard si les pays colonisés avaient utilisé le discours identitaire pour mobiliser « les damnés de la terre ».

Cette résistance a été faite à travers la réhabilitation des sources et des textes fondateurs de l'islam. Les réformistes, malgré leur usage stratégique de la modernité, ne se sont pas laissés entièrement envoûter par ses attraits car ils ont refusé de sacrifier la pensée ancestrale au profit d'une pensée importée et imposée par les empires européens. Même si elle rejoint l'antimodernité européenne dans certaines de ses options, la contremodernité arabe s'en distingue à bien des égards. Les penseurs de cette contre-modernité visent à penser leur société, leur histoire et leur culture par rapport à des puissances dominantes qui aliénaient le sujet colonisé en le dépossédant de son passé culturel. Il est donc question de repenser les cultures soumises au joug des puissances impériales en tant que forces exogènes, alors que les antimodernes européens résistaient à la modernité des Lumières en tant que phénomène historique endogène ${ }^{4}$.

Le rapport à la modernité occidentale a fait naître, dans le monde arabe en général, un sentiment d'appartenance à la "nation arabe » qui a pu se consolider progressivement, sans vraiment constituer une masse homogène. Ce sentiment a commencé à se former politiquement vers la fin du XIX et le début du $\mathrm{XX}^{\mathrm{e}}$ siècles en Syrie et au Liban face à l'empire ottoman à travers la lutte contre ce qu'on appelait à l'époque "la turquisation» (tatrik) de la Grande Syrie. Le grand récit de l'unité arabe est né dans cette lutte qui s'était déplacée, après l'affaiblissement de l'empire ottoman, vers un autre danger : l'expansionnisme britannique et français. Nous pouvons dire, et cela a été suggéré par Hannah Arendt dans ses analyses consacrées à l'impérialisme ${ }^{5}$, que les empires coloniaux ont éveillé et ravivé la conscience nationale arabe. Ce sont les islamistes qui ont pris le relais partout dans le monde arabo-islamique en tirant profit de la désaffection des populations vis-à-vis des réformateurs et des libéraux qui n'ont pas pu réaliser les différentes promesses de progrès et de prospérité

4. À propos de l'idéologie antimoderne européenne, voir l'ouvrage d'Antoine Compagnon, Les Antimodernes, Paris, Gallimard, 2006.

5. L'Impérialisme, dans Les Origines du totalitarisme, Paris, Quarto/Gallimard, 2002, p. 372. (Réédition : Seuil, 2006). 
faites aux populations arabes ${ }^{6}$. Les islamistes les plus radicaux (qu'on connaissait surtout sous le nom de Frères musulmans et de salafistes) prônaient la rupture avec l'Occident en le stigmatisant et en en faisant un ennemi de l'islam et un allié inconditionnel de l'État d'Israël, surtout depuis l'expulsion des Palestiniens de leurs territoires en 1948.

\section{Modernité littéraire}

La modernité littéraire a été introduite au Maroc par l'empire colonial français et les oeuvres publiées en arabe au Machrek (l'Orient arabe). D’où la dépendance des écrivains marocains vis-à-vis de deux instances de consécration: la France et l'Orient arabe, notamment l'Égypte et le Liban. La fameuse mission civilisatrice de la France a commencé par l'instruction, la formation et la valorisation d'une élite locale qui allait par la suite reproduire (certes avec des nuances) la version abstraite du discours universaliste des Lumières. Les liens avec les pays arabes d'Orient ont permis à l'élite arabophone marocaine (qui entretenait une relation ambiguë avec la mission civilisatrice du monde occidental) d'accéder aux narrations modernes à travers les traductions des textes occidentaux en arabe (à vrai dire l'arabisation et l'égyptianisation qui sont souvent des adaptations et rarement des traductions comme on peut le remarquer chez un écrivain aussi remarquable que Al-Manfaluti) et les mouvements poétiques qui célébraient le vers libre. Le roman européen a connu une large diffusion dans le monde arabe grâce aux traductions, alors que la modernité poétique venait directement du Liban à travers la revue littéraire Chi'r (Poésie) dirigée par le poète Adonis qui se plaît à s'identifier en tant que syro-libanais.

Les écrivains de langue française élaborent conjointement une contreécriture de leur patrimoine culturel et de l'héritage culturel occidental; ce que le regretté Abdelkébir Khatibi appelle la «double critique ${ }^{7} »$. En revanche, les écrivains de langue arabe, se définissent d'abord comme écrivains arabes car le panarabisme culturel reste encore déterminant dans leur appartenance au champ littéraire. Être publié et consacré en Égypte ou au Liban a toujours été considéré comme un acte valorisant, au moins quand un écrivain de langue arabe commence sa carrière littéraire. Au Maroc, les cas d'Abdekrim Ghallab, Abdallah Laroui, Salem Himmich et d'autres sont révélateurs de cette situation de dépendance à l'égard du champ littéraire du Machrek. En 2002 Hamid Lahmidani a obtenu le Prix du Roman Arabe en Jordanie pour son roman Rihlatun kharija al-tariqi al-sayyar ${ }^{8}$ (Voyage hors autoroute), alors que ce texte est passé presque inaperçu au

6. Pour plus de détails, voir Ali Oumlil, Mawaqifal-Fikr al-Arabi mina al-Taghyiratal-Dawliya : al-Dimuqratiya wa al-'Awlama (Positions de la pensée arabe face aux changements internationaux : démocratie et mondialisation), Oman, Muntada al-fikr al-arabi, 1998.

7. Maghreb Pluriel, Paris, Denoël, 1983.

8. Meknès, Publications Alamat, 2000 (en arabe). 
Maroc. Il en va de même pour Hamid Ben Hadouga, Tahar Ouattar et Wassini Laredj en Algérie. Messaadi en Tunisie et Ibdahim Al-Kouni en Libye constituent des voix majeures de la littérature arabophone.

Le Maghreb est incontestablement l'aire géographique qui a vécu les tensions les plus fortes entre écrivains francophones et écrivains arabophones après les indépendances. Les institutions politicodiplomatiques françaises ont toujours suivi avec beaucoup d'intérêt ces tensions et ont adopté des stratégies de « présence culturelle » qui relèvent souvent d'une stratégie concurrentielle avec les cultures de langue arabe. Les services culturels français alimentent et maintiennent ces tensions en encourageant l'élite francophone au détriment de l'élite arabophone. Ayant compris le désir hégémonique de la France sur leurs scènes culturelles, la monarchie marocaine et la présidence tunisienne ont adopté une stratégie d'encouragement de l'élite francophone, en tant qu'élite considérée comme "porteuse de valeurs modernes ", tout en dressant contre elle, quand le besoin s'en ressentait, l'élite arabophone. Cette stratégie n'aurait certainement pas eu de succès si ces deux élites étaient majoritairement bilingues et autonomes sur le plan intellectuel. L'antagonisme a été possible car les enjeux de légitimation nationaliste et anticolonialiste passaient essentiellement par (outre l'intégrité territoriale) l'islam et la langue arabe. Ces deux derniers éléments n'ont certes pas toujours été liés, mais la consolidation de la nation musulmane à l'époque abbaside a conduit à un amalgame de la langue arabe et de l'islam (ce qui, dès la naissance de l'impérialisme européen, a toujours arrangé une partie majoritaire de l'élite occidentale) qui a fini par semer la confusion entre la dimension religieuse et le substrat linguistique. La guerre des langues a été profitable au maintien de l'hégémonie culturelle et linguistique française au Maghreb. En échange de cette complicité avec la politique culturelle française, la monarchie marocaine et la présidence tunisienne ont toujours exigé (et obtenu) des compromissions de la part de la France sur les questions des droits de l'homme.

L'Algérie, pour des raisons liées à la légitimation de son élite politique, ne s'inscrit pas dans cette même logique de servilité culturelle. Sa situation est peut-être plus complexe et moins facile à circonscrire du fait de sa forte ambivalence culturelle. Les Algériens sont plus acculturés que leurs voisins marocains et tunisiens, mais en même temps ils sont plus virulents dans leurs critiques de l'hégémonie française. Deux éléments font écran à toute possibilité de négociation sereine entre l'Algérie et la France : d'un côté, le paradigme du Moudjahid algérien et, de l'autre, le désir impérial français. Par conséquent, l'implantation institutionnelle de la France est plus faible en Algérie que dans les deux autres États du Maghreb francophone. En revanche, à cause précisément de ces tensions et de la formation linguistique de l'élite algérienne, c'est cette dernière qui a produit le 
plus de textes littéraires francophones par rapport à ses voisins aussi bien quantitativement que qualitativement.

Il a cependant été d'usage de considérer les auteurs issus du Maghreb comme faisant partie d'un seul et même champ littéraire. Le regretté Jean Déjeux (qui a surtout le mérite d'avoir établi des bibliographies très riches sur les auteurs du Maghreb) a intitulé, à juste titre, son ouvrage le plus important Maghreb. Littératures de langue française ${ }^{9}$. Le pluriel est ici nécessaire parce que, malgré les questionnements communs aux littératures francophones algérienne, marocaine et tunisienne, il y a des particularités thématiques et historiques à chacune d'entre elles, sans oublier le fonctionnement de chaque champ littéraire indépendamment de l'autre. La fiction d'un « champ littéraire maghrébin » participe certainement d'une volonté d'écrire une histoire commune, mais elle se heurte aux réalités des textes qui dessinent une autre cartographie littéraire du Maghreb. L'unité politicoéconomique, plus que jamais nécessaire, n'implique pas forcément l'invention d'un champ littéraire commun. Trois textes ont marqué la modernité des littératures postcoloniales du Maghreb : La Statue de sel d'Albert Memmi, Le passé simple de Driss Chraïbi et Nedjma de Kateb Yacine ${ }^{10}$ qu'on peut considérer comme le véritable début du roman moderne au Maghreb. C'est dire que les écrivains maghrébins arabophones sont venus plus tard à ce genre littéraire qui était déjà introduit en Orient arabe à travers des traductions/adaptations. Dafanna al-Madî (Le Passé enterré) du Marocain Abdelkrim Ghallab et Rîhu al-Janoub (Le Vent du Sud) de l'Algérien Abdelhamid Ben Hadouga sont les premiers romans marocain et algérien écrits en arabe, publiés respectivement en 1966 et 1971.

Si le versant littéraire marocain écrit en français date du protectorat, il en est autrement des textes littéraires en langue arabe. La poésie et la littérature de voyage qui était généralement l'apanage des diplomates marocains, comme nous l'avons vu plus haut, ont existé en langue arabe bien avant le protectorat avec des poètes comme Abdelkrim Ben Zakour (Assirâj alwahhâj fi Madhi Sahib al-Taj wa al-Mi'raj (Le Cierge lumineux pour l'éloge de l'homme de la Couronne et de l'Ascension), Ibn Taïb Al-Alami et Ibn Idriss ainsi que des voyageurs tels Ibn Battouta, Mohammed Al-Rhassani Al-Andaloussi et Mohammed Ben Othman Al-Meknassi, pour ne citer que ceux-là. Mais aussi bien la poésie que les récits de voyage étaient écrits dans un style codifié selon les règles de la rhétorique arabe classique.

Sous le protectorat, le mouvement poétique s'était bien développé bien qu'il fût engagé dans un mimétisme du patrimoine arabe classique et surtout du patrimoine abbasside et andalou comme c'est le cas chez les poètes Thami Mdarhri et Al Haj Driss ben Ali. À cette même période, une poésie marocaine engagée prendra naissance avec Ayna Alliwa' (Où est

9. Paris, Arcantère, 1993.

10. Respectivement : Corréa, 1953; Denoël, 1954 et Seuil, 1956. 
l'étendard?), poème composé par Mohamed Ben Abdallah El Othmani Soussi après que les autorités françaises avaient enlevé le drapeau marocain des administrations makhzéniennes ${ }^{11}$ du Souss, Allal Al Fassi, Mokhtar Soussi et Mohamed El Mahdi El Hajoui qui lient langue arabe et unité nationale, sans oublier Mohamed El-Yamani Nassiri qui fait le panégyrique de Ben Abdelkrim au moment de la résistance aux forces françaises et espagnoles. Abderrahman Ben Zidane représente plutôt un mouvement poétique d'inspiration religieuse comme en témoigne son recueil de poésie Annour Allâih bi Mawlid al-Rassoul al-Khatam al-Fatih (La Lumière projetée en hommage à la naissance du sceau des prophètes). Les formes narratives arabophones les plus proches des techniques du roman sont peut-être la relation de voyage publiée par Ibn Al-Moaqat en 1933 sous le titre Al-rihla al-Mourrakouchiya (Le Voyage de Marrakech) et le récit intitulé Al-Zaouiya (Le Sanctuaire) que Thami El-Ouazzani avait publié en $1942^{12}$.

Au début de la seconde moitié du $\mathrm{xx}^{\mathrm{e}}$ siècle (1954), Driss Chraïbi fait son entrée dans le monde littéraire avec son roman incisif Le Passé simple $^{13}$. À cette époque, il y avait seulement deux auteurs francophones marocains : Abdelkader Chatt qui a publié Mosaïques ternies à Paris en 1932 et Ahmed Sefrioui qui a publié en 1949 Le Chapelet d'ambre chez Julliard et plus tard, en 1954, La Boîte à merveilles aux éditions du Seuil. Quand on examine ces textes, on arrive à la conclusion que la naissance du roman marocain est liée à une conscience aiguë du balancement entre les valeurs du passé (la tradition) et les nécessités du présent (la modernité) que ces trois auteurs incarnent par excellence à travers leurs fictions. Ce balancement a donné lieu à un tiers espace qui est passé par une double critique. Cet entre-deux a été négativement perçu au moment de la décolonisation et ne sera valorisé qu'à partir des années 1980. Le Passé simple illustre parfaitement cette difficulté de dire sa subjectivité dans un contexte de décolonisation.

En effet, avec ce roman, Driss Chraïbi, jeune marocain issu de la bourgeoisie musulmane contre laquelle il se révolte par esprit de réforme et non de dénigrement, fait une entrée fracassante dans le monde littéraire francophone. Son roman a été publié à un moment où le Maroc préparait son indépendance. C'est ce qui explique, en partie, sa réception défavorable par les nationalistes marocains, surtout par un journaliste de Démocratie qui avait accusé l'auteur de trahison. Driss Chraïbi a renié par la suite son livre en publiant dans le même journal un article intitulé « Je renie

11. Les autorités marocaines qui dépendent du Sultan.

12. Voir à ce propos Abbas Al-Jirari, Al-Adab Al-Maghribî, t. 1, Rabat, Maktabat alMa'ârif, 1979.

13. Driss Chraïbi, Le Passé simple, Paris, Denoël, 1954 (avec plusieurs rééditions dans la collection « Folio » de Gallimard depuis 1985). 
Le Passé simple ». L'auteur y a plutôt expliqué sa position par rapport au contenu de son livre dont le but était de secouer les mentalités sclérosées qui érigent l'autoritarisme en principe de vie. Que raconte donc ce roman qui a été réhabilité, dix ans après sa publication, par le poète marocain Abdellatif Laâbi dans la revue Souffles?

Ce récit fondateur de la modernité romanesque au Maroc est construit selon un processus expérimental. Dès le premier chapitre intitulé «Les éléments de base ", le protagoniste Driss Ferdi vit la crise du passage d'un enseignement traditionnel, représenté par l'école coranique, à un enseignement moderne, représenté par l'école française. Le premier système d'enseignement est disqualifié à cause de sa perversion et de son excès d'autoritarisme, alors que le second système incarne, pour le narrateur, l'ouverture et l'autonomie. La critique de la société traditionnelle est corroborée par la figure négative du père, appelé le Seigneur, et la soumission inconditionnelle de la mère qui connaîtra un sort lugubre à la fin du récit puisqu'elle va se suicider. Dès le premier chapitre, le conflit entre Driss et son père est mis en scène pour donner le ton au récit :

\footnotetext{
Il m'attira à lui, me reposa. Entre les deux gestes, je ne vis que des incisives en or. L'horloge sonna la demie. Sur ma figure coulait le crachat ${ }^{14}$.
}

Le père castrateur détenteur d'un pouvoir polymorphe prend une proportion importante dans ce récit qui influencera la postérité littéraire au Maghreb comme en témoigne, entre autres romans, La Répudiation de Rachid Boudjedra publié en $1969^{15}$ ou, plus récemment Noces et Funérailles de Karim Nasseri ${ }^{16}$.

Le chapitre suivant qui a pour titre « Période de transition » est, du point de vue narratif, un lieu de transit : le conflit entre père et fils y est laissé de côté pour produire un discours sur la ville de Fès que le narrateur appelle «cité des Seigneurs ${ }^{17}$ ». Driss accompagne sa mère à Fès pour que la protection des saints de cette ville couvre les affaires juteuses du père : la sainteté au service du marketing du Seigneur! Cette ville est pour lui un catalyseur de souvenirs où la dysphorie l'emporte largement sur l'euphorie bien qu'il y goûte aux plaisirs de la liberté. Driss, pour qui cette ville humide porte les traces d'un passé haï, nous dit : «Je n'aime pas cette ville. Elle est mon passé et je n'aime pas mon passé ${ }^{18} »$. Elle est détestée car le double du père y demeure : c'est le $f q i h^{19} \mathrm{Si}$ Kettani qui ressemble, à travers des signes sociaux, au père de Driss. En effet, ils sont tous les deux

14. Driss Chraïbi, op. cit., p. 30.

15. Rachid Boudjedra, La Répudiation, Paris, Denoël, 1969.

16. Karim Nasseri, Noces et Funérailles, Paris, Denoël, 2001.

17. Driss Chraïbi, op. cit., p. 69.

18. Ibid.

19. Le Maître de l'école coranique. 
Hajs $^{20}$, riches, puissants, honorables et honorés ${ }^{21}$. À travers la figure du fqih, c'est toute une grammaire socio-religieuse qui est déconstruite car elle est productrice d'une syntaxe de l'hypocrisie : le fqih cache sa pédophilie et sa corruption sous les apparences du sacré.

Les chapitres intitulés respectivement «Le réactif» et «Le catalyseur » permettent la mise en texte des facteurs qui font avancer rapidement le tempo de l'histoire narrée. La crise y atteint son paroxysme et la rupture entre Driss Ferdi et son père y est déjà consommée. Le père est ruiné et le frère du narrateur, Hamid, décède. C'est tout un monde auquel le personnage-narrateur a été soumis et contre lequel il n'a cessé de se positionner qui s'écroule. La « haine » qui loge dans les tripes de Driss trouve enfin satisfaction. Mais comme le meurtre du Père est lourd de conséquences, la délivrance se double d'un tiraillement identitaire et la fuite semble être une solution factice et inefficace pour lutter contre la sclérose de la société marocaine dont Driss est issu. C'est le moment où, dans les littératures du Maghreb, émerge le thème de l'acculturation qui est la conséquence logique de ce tiraillement identitaire entre le même et l'autre. L'idée matricielle du Passé Simple a été formulée en quelques mots par le narrateur vers la fin de son récit :

\footnotetext{
Un roman. Un roman, entends-tu? Dont les éléments seraient : une histoire de thés, un bref séjour à Fès, la mort d'Hamid, ma révolte ${ }^{22}$.
}

Dans le dernier chapitre intitulé « Les éléments de synthèse », Driss largue les amarres et annonce son départ en France pour « puiser dans les stocks de réformes sociales » et autres mouvements présents dans le marché des biens symboliques de l'Occident. L'essentiel pour lui est d'éviter la «bonne vieille résignation » que lui propose sa société.

Le protagoniste est en quête d'une alternative à sa situation de subalterne. Mais cette quête est structurée par un processus d'acculturation qui sépare le personnage, et par ricochet l'écrivain, de sa culture et de sa langue maternelle. Le déchirement, lié à la conjoncture historique du colonialisme, était traumatique pour les écrivains qui furent à la charnière de la colonisation et de la décolonisation. Cette situation qui était très intense chez beaucoup d'écrivains francophones, entre les années 1950 et 1970, a perduré jusqu'au début des années 1980.

L'élite intellectuelle marocaine, qu'elle soit traditionaliste ou moderniste, a toujours lutté contre l'idée de la modernité comme triomphe de l'universel sur le particulier. Elle a considéré que les missions civilisatrices n'étaient que des prétextes, parmi tant d'autres, pour légitimer le colonialisme qui a contribué à introduire une modernité « bancale » dans le

20. Musulman qui a accompli le pèlerinage à la Mecque.

21. Ibid., p. 79.

22. Driss Chraïbi, op. cit., p. 187. 
monde arabe en général, et au Maroc en particulier. Ce n'était par ailleurs pas un hasard si les nationalistes marocains avaient utilisé le discours identitaire pour mobiliser les masses populaires. On peut cependant dire, avec la distance qui est la nôtre aujourd'hui, que la défense chauvine de la tradition culturelle locale est un piège qui a conduit à l'affirmation exacerbée des identités car celles-ci, dans un tel cas, ne pouvaient être définies que par opposition à l'autre (souvent réduit à un autrui stéréotypé).

Ce qui caractérise la modernité marocaine d'aujourd'hui, c'est le phénomène de contamination culturelle qui abolit les critères traditionnels de distance et de nette séparation entre les différentes cultures « majeures » qui constituent le Maroc comme " multiplicité » (arabe, berbère, juive et occidentale). Cela ne signifie pas que ces cultures tendent à s'accepter et à communiquer sereinement. Cela signifie qu'elles se trouvent dans l'obligation de négocier leurs différences pour trouver des compromis qui rendent le vivre-ensemble possible. Cette situation a eu des répercussions indéniables sur la littérature qui, depuis Driss Chraibi, est entrée dans le paradigme de la modernité littéraire par l'usage du genre romanesque et la déstructuration du dispositif énonciatif mimétique.

Khalid Zekri

Université de Meknès/Centre de recherches francophones de Leipzig 